\title{
Unpredicted spontaneous extrusion of a renal calculus in an adult male with spina bifida and paraplegia: report of a misdiagnosis. Measures to be taken to reduce urological errors in spinal cord injury patients Subramanian Vaidyanathan*1, Peter L Hughes ${ }^{2}$, Bhakul M Soni1, Gurpreet Singh ${ }^{1}$, Paul Mansour ${ }^{3}$ and Pradipkumar Sett ${ }^{1}$
}

\begin{abstract}
Address: ${ }^{1}$ Regional Spinal Injuries Centre, District General Hospital, Southport PR8 6PN, UK, ${ }^{2}$ Department of Radiology, District General Hospital, Southport PR8 6PN, UK and ${ }^{3}$ Department of Cellular Pathology, District General Hospital, Southport PR8 6PN, UK

E-mail: Subramanian Vaidyanathan* - vaidyanathansiu@ hotmail.com; Peter L Hughes - Peter.Hughes@ mail.soh-tr.nwest.nhs.uk; Bhakul M Soni - Bakul.Soni@mail.soh-tr.nwest.nhs.uk; Gurpreet Singh - gurpreet.singh@ukgateway.net;

Paul Mansour - Paul.Mansour@mail.soh-tr.nwest.nhs.uk; Pradipkumar Sett - Pradip.Sett@mail.soh-tr.nwest.nhs.uk

${ }^{*}$ Corresponding author
\end{abstract}

This article is available from: http://www.biomedcentral.com/I47/-2490/I/3

(C) 200I Vaidyanathan et al; licensee BioMed Central Ltd. Verbatim copying and redistribution of this article are permitted in any medium for any noncommercial purpose, provided this notice is preserved along with the article's original URL. For commercial use, contact info@biomedcentral.com

\begin{abstract}
Background: A delay in diagnosis or a misdiagnosis may occur in patients with spinal cord injury $(\mathrm{SCl})$ or spinal bifida as typical symptoms of a clinical condition may be absent because of their neurological impairment.
\end{abstract}

Case presentation: A 29-year old male, who was born with spina bifida and hydrocephalus, became unwell and developed a swelling and large red mark in his left loin eighteen months ago. Pyonephrosis or perinephric abscess was suspected. X-ray of the abdomen showed left-sided staghorn calculus. Since ultrasound scan showed no features of pyonephrosis or perinephric abscess, he was prescribed a prolonged course of antibiotics for infection presumed to arise from the site of metal implant in spine. He developed a discharging sinus, following which the loin swelling and red mark subsided. About three months ago, he again developed a red mark and minimal swelling in the left loin. Ultrasound scan detected no abnormality in the renal or perinephric region. Therefore, the red mark and swelling were attributed to pressure from the backrest of his chair. Five weeks later, the swelling in the left loin burst open and a large stone was extruded spontaneously. An X-ray of the abdomen showed that he had extruded the central portion of the staghorn calculus from left kidney. With hindsight, the extruded renal calculus could be seen lying in the subcutaneous tissue of left loin lateral to the $10^{\text {th }}$ rib in the X-ray of abdomen, which was taken when he presented with red mark and minimal swelling.

Conclusion: This case illustrates how mistakes in diagnosis could occur in spinal cord injury patients, and highlights the need for corrective measures to reduce urological errors in these patients. Voluntary reporting of urological errors is recommended to facilitate learning from our mistakes. In the patients who have marked spinal curvature, ultrasonography of kidneys and perinephric region may not be entirely reliable. As clinical symptoms and signs may be non-specific in $\mathrm{SCl}$ patients, they require prompt, detailed and occasionally, repeated investigations. A joint team approach by health professionals belonging to various medical disciplines, which is strengthened by frequent, informal and honest discussions of a patient's clinical condition, is likely to reduce urological errors in $\mathrm{SCl}$ patients. 


\section{Background}

A delay in diagnosis or a misdiagnosis may occur in the patients with spinal cord injury (SCI) or spinal bifida as typical symptoms of a clinical condition may be absent in these patients because of their neurological impairment. [1]. Physicians may commit an error in diagnosis of a clinical condition as the symptoms and signs may be non-specific [2], or mistakes could occur during interpretation of medical images or histological appearances. Atypical decubital fibroplasia [3], which is a unique type of pressure sore displaying degenerative and regenerative features distinct from decubitus ulcer, may be misdiagnosed by pathologists and clinicians as a sarcoma. Epidemiology of medical errors showed that patients aged over 64 years have a greater risk of serious injury from adverse events than younger patients. [4]. We believe that patients with spinal cord injury and spina bifida should be included in the high-risk group for possible occurrence of medical errors. We report an adult with spinal bifida and paraplegia, who presented with redness and minimal swelling in the loin. This was diagnosed initially as a pressure mark. Subsequently, a large renal calculus was extruded out of the loin swelling spontaneously.

\section{Case presentation Case report}

A 29-year old male, who was born with spina bifida and hydrocephalus, presented recently with recurrent episodes of redness and minimal swelling in the left loin. At the age of eight years, he had an ileal conduit urinary diversion and surgery for kyphoscoliosis. An intravenous pyelography performed five years ago showed bilateral renal calculi (left greater than right). Right kidney was functioning, but no contrast was seen in the left pelvicalyceal system or ureter. Eighteen months ago, he became unwell and developed a swelling and large red mark in left loin. Pyonephrosis or perinephric abscess was suspected. X-ray of abdomen showed left-sided staghorn calculus. There was a small stone in the right kidney. (Figure 1). Ultrasonography revealed no evidence of hydronephrosis. Since ultrasound scan showed no features of pyonephrosis or perinephric abscess, he was prescribed prolonged course of antibiotics for infection presumed to arise from the site of metal implant in spine. He developed a discharging sinus following which, the loin swelling and red mark subsided.

About three months ago, he again developed a red mark and minimal swelling in the left loin. He did not have fever. Blood tests were not done during this visit. However, blood tests were performed five days later. The results were as follow: Haemoglobin: $7.0 \mathrm{~g} / \mathrm{dL}$; White cell count: 12.6 x 109/L; Neutrophils: 10.37 x 109/L. Urea: 14.2 $\mathrm{mmol} / \mathrm{L}$; Creatinine: $199 \mathrm{umol} / \mathrm{L}$; C-reactive protein (CRP): $171.4 \mathrm{mg} / \mathrm{l}$. In view of elevated white cell count

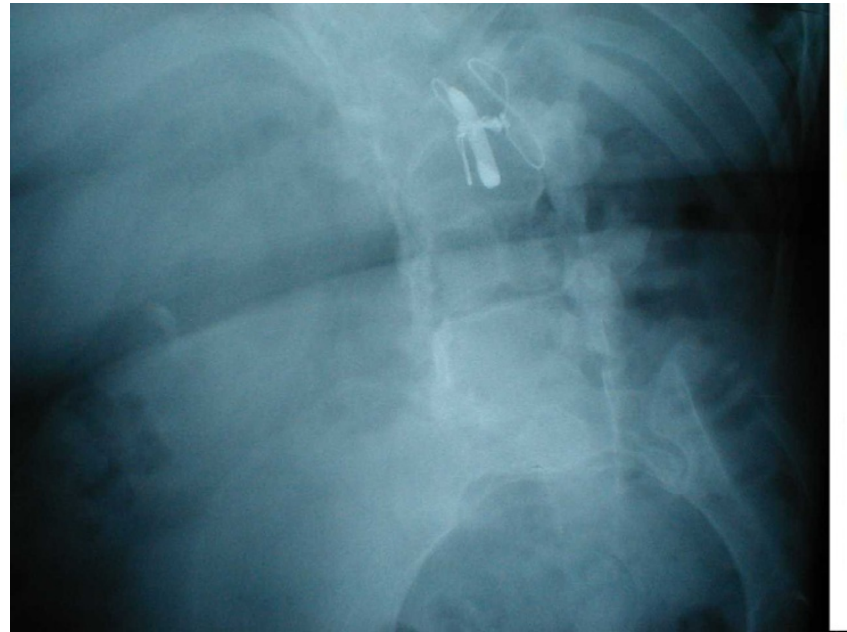

Figure I

X-ray of abdomen (05052000): This X-ray of abdomen shows large staghorn calculus in the left kidney and a small stone in right kidney.

and high CRP level, the general practitioner was requested to prescribe antibiotic. Patient received amoxicillin 250 mg every eight hours by mouth. X-ray abdomen showed left-sided staghorn calculus. (Figures 2 and 3). Ultrasonography showed no evidence of hydronephrosis. Since ultrasound scan detected no abnormality in the renal or perinephric region, the red mark and swelling were attributed to pressure from backrest of his chair. About five weeks after the radiological examination, the swelling in left loin burst open and a large stone was extruded spontaneously. (Figure 4). Following spontaneous extrusion of renal calculus, the sinus became smaller. (Figure 5). There was minimal discharge of seropurulent fluid. An X-ray of abdomen showed that he had extruded central portion of the staghorn calculus from left kidney. (Figure 6). With hindsight, the extruded renal calculus could be seen lying in the subcutaneous tissue of left loin lateral to the $10^{\text {th }}$ rib in the X-ray of abdomen, which was taken when he presented with red mark and minimal swelling (Figures 2 and 3 ). This radio opaque density situated in the soft tissue of left loin was not present in either of the X-ray of abdomen taken earlier (Figure 1) or subsequent to extrusion of the renal calculus. (Figure 6).

\section{Discussion}

Breatnach and associates [5] reported spontaneous extrusion of a staghorn calculus into the flank soft tissues, which was diagnosed by CT. The stone was demonstrable on CT as fragments of calciflc density appearing in the subcutaneous area. Breatnach and associates [5] stated that such a complication of renal calculus disease has not been reported previously. We failed to detect the calculus lying in the soft tissue of left loin in an abdominal X-ray 


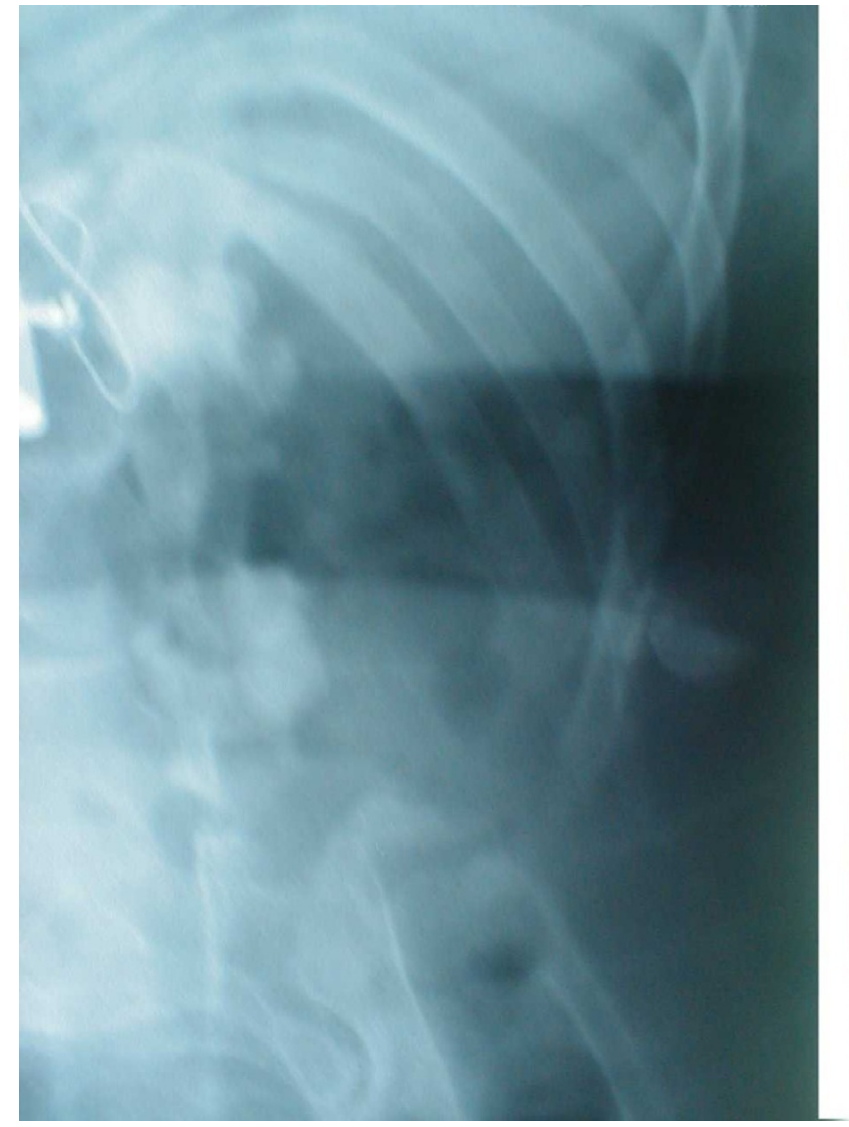

\section{Figure 2}

X-ray of abdomen (1010200I) Abdominal X-ray shows leftsided upper and lower pole staghorn calculi with defect in the central portion.

(Figure 3), when this patient presented with redness and swelling in left loin. The ultrasound scan showed no abnormality in the kidney or perinephric region. Unfortunately, we did not evaluate the loin wall during this ultrasound examination. We attributed the swelling and redness to pressure caused by backrest of his chair, since SCI patients are highly susceptible to develop pressure sores. Instead of making a presumptive diagnosis of pressure mark, we should have persevered in our diagnostic efforts and should have reviewed the X-rays of abdomen, which were taken at different times. Obviously, there was an over reliance on the ultrasound scans. In hindsight, we realise that should have performed a CT of abdomen. Had we carried out a CT of abdomen, we would have detected the extruded stone lying in the subcutaneous tissue, and reached the correct diagnosis of spontaneous extrusion of renal calculus.

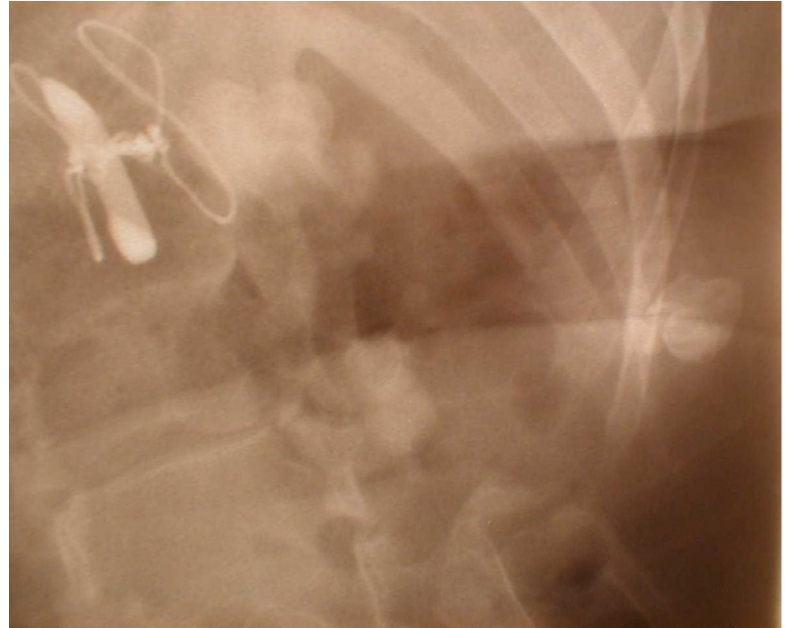

Figure 3

A close-up view of left lumbar region in the X-ray of abdomen A large stone is seen lying in the soft tissue of left loin lateral to the $10^{\text {th }}$ rib.

\section{What should we do to reduce urological errors in the pa- tients with spinal cord injury?}

Some pertinent issues for minimising errors in spinal cord injury medicine are listed below.

Voluntary reporting of urological errors will be helpful to facilitate learning from our mistakes or near miss: A recent report from the Institute of Medicine, To Err is $\mathrm{Hu}$ man, strongly recommends complementary mandatory incident reporting systems and voluntary near miss reporting systems in health care. [6]

SCI patients require prompt, detailed and if necessary, repeated investigations as clinical symptoms and signs may be non-specific in SCI patients. Absence or paucity of typical symptoms and signs in SCI patients is well illustrated by this case. This paraplegic patient, who developed perinephric abscess and extruded central portion of a staghorn calculus, did not develop loin pain at all.

SCI patients are susceptible to develop certain clinical conditions and diagnosis of these disorders may not be easy. A few examples pertaining to urinary tract of SCI patients are given below:

- In SCI patients, renal calculi may be obscured by bowel gas shadows or by loaded colon in a plain X-ray of abdomen. Ultrasonography of kidneys and perinephric region may be unreliable in SCI patients who have marked spinal curvature. Even CT may be distorted by the patient's spinal and pelvic deformity and true axial images may not be obtained; this can make definition of soft tissue planes difficult. 


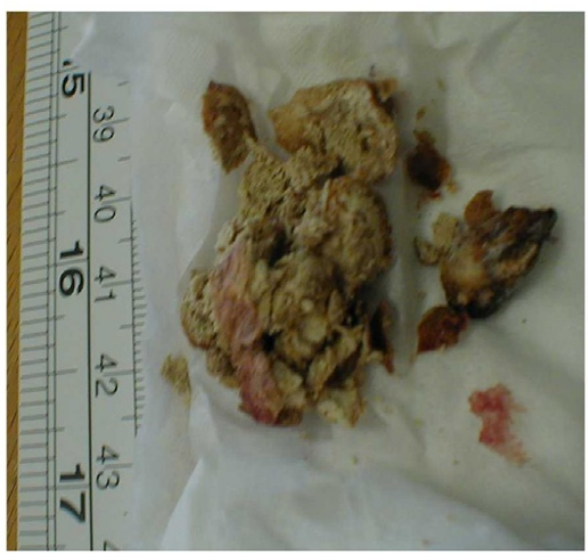

\section{Figure 4}

Photograph of the stone Renal calculus, which was extruded spontaneously through the loin, is shown.

- SCI patients are at increased risk for developing bladder cancer. When vesical malignancy occurs, SCI patients may not always present with the classical symptom of haematuria. Cystoscopy, when performed to screen for squamous cell cancer of the bladder in spinal cord injured patients with chronic or recurrent urinary tract infection, has been shown to result in an earlier stage at diagnosis and convey a survival advantage. [7]

- Histological interpretation of bladder biopsies in SCI patients may be difficult because of associated inflammatory changes. Immunohistochemistry of urinary bladder biopsy, in addition to routine haematoxylin \& eosin staining, may be a useful diagnostic aid in selected SCI patients. Immunostaining with cytokeratin 14 may help in early detection of squamous metaplasia.

Immunostaining with Cytokeratin 20 is likely to facilitate the diagnosis of urothelial dysplasia. [8]

- When the result of a diagnostic procedure is at variance with the overall clinical probability, a repeat investigation might be useful, particularly in SCI patients, as these patients do not manifest typical symptoms and signs. A patient, who had sustained spinal cord injury and paraplegia 36 years ago, presented with recurrent urinary infection. He did not give a history of haematuria. A bladder biopsy showed features of inflammation and papillary cystitis. There was no evidence of dysplasia or neoplasia in this biopsy. (Figure 7). However, a repeat bladder biopsy, which was performed three weeks later, revealed squamous cell carcinoma. (Figure 8).

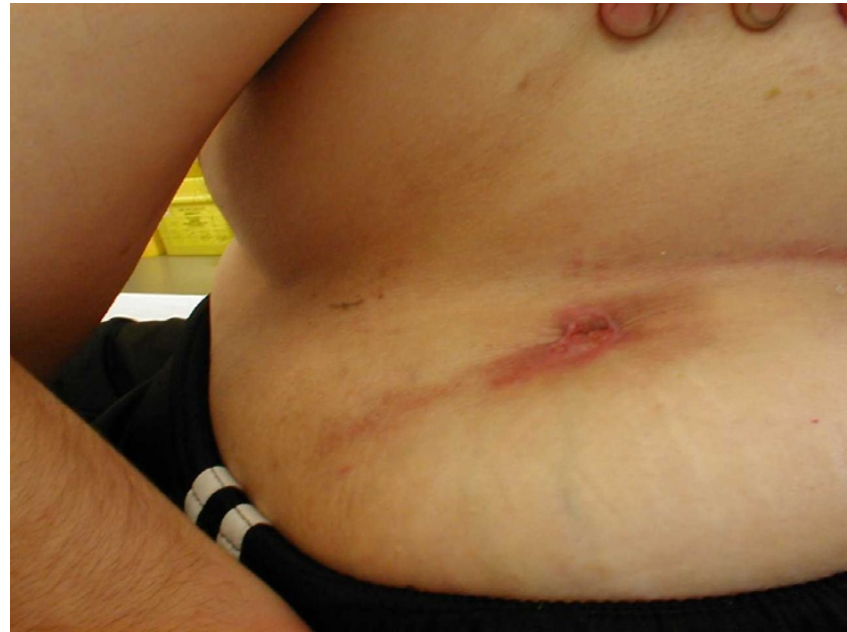

\section{Figure 5}

Clinical photograph of left loin This photograph shows a sinus in left loin through which the central portion of a staghorn calculus was extruded spontaneously.

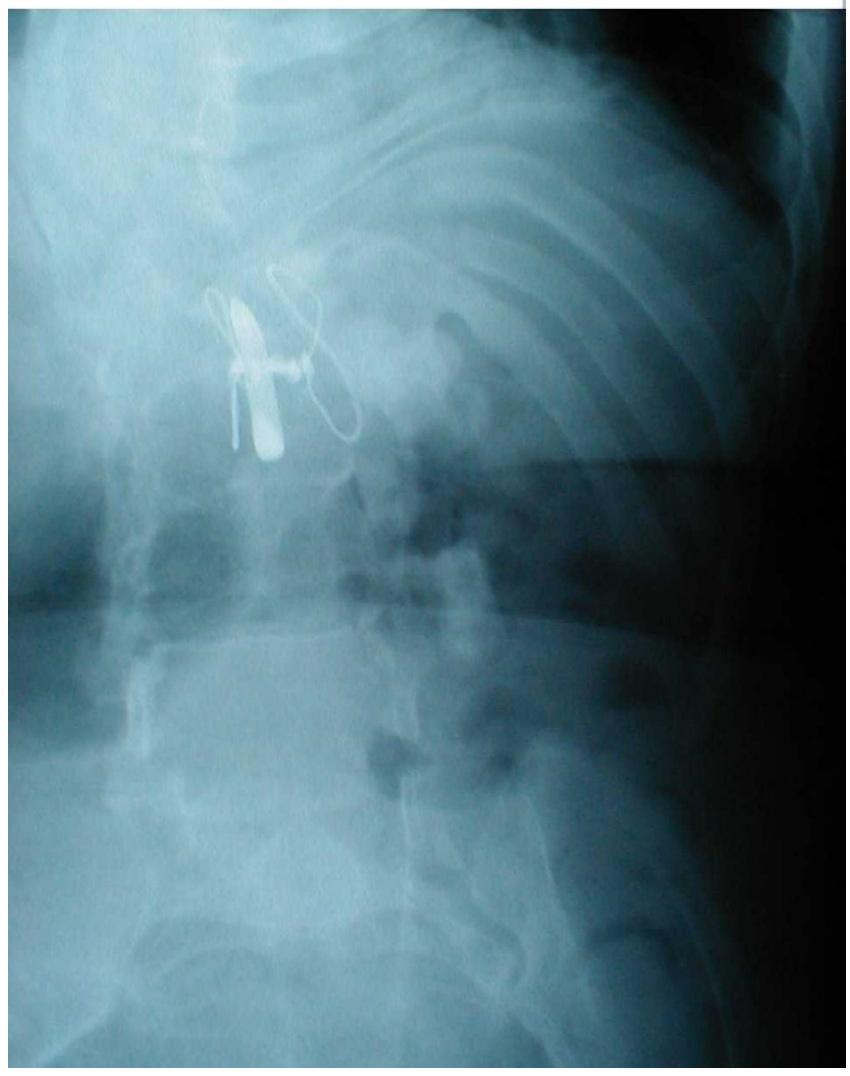

Figure 6

X-ray of abdomen ( $|3||200|)$ This abdominal X-ray shows the radio opaque shadow previously located in left loin is no longer present. 


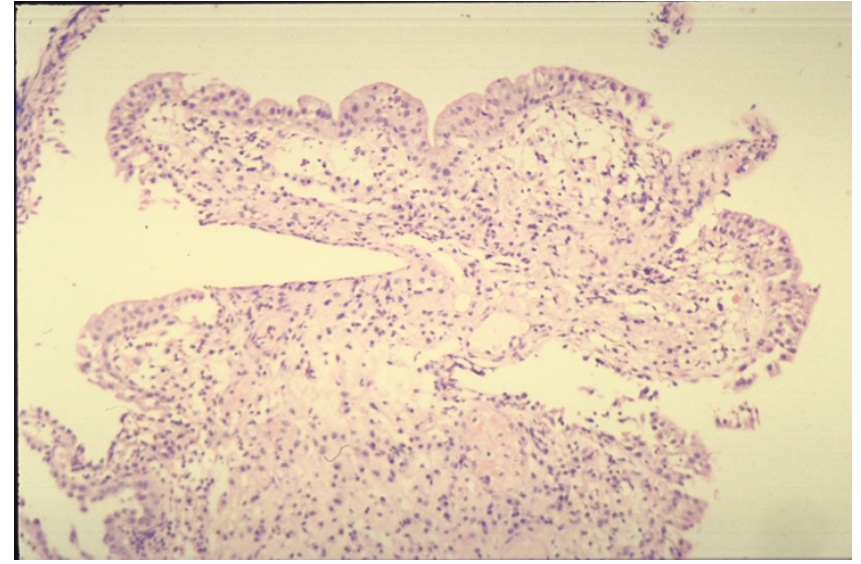

Figure 7

A male patient sustained spinal cord injury and paraplegia 36 years ago. He presented with recurrent urinary infection. There was no history ofhaematuria. Histology of bladder biopsy shows moderately inflamed bladder mucosa and papillary cystitis. There was no evidence of dysplasia or neoplasia in this biopsy. (HPOI/06270)

SCI patients may develop clinical problems, which affect different organ systems and require investigations by physicians with expertise in their specialities, e.g. imaging studies by radiologists, histopathological examination of biopsies, gastrointestinal or, urinary tract endoscopy. Therefore, health professionals working in various disciplines should remove artificial barriers and hierarchical settings, which exist to varying degrees in healthcare system, and hold frequent, informal and honest discussions of a SCI patient's clinical condition. Such a joint team approach in reaching a diagnosis, and in implementing a treatment regime, is likely to reduce medical errors in SCI patients.

\section{Conclusions}

SCI patients require detailed and sometimes, repeated investigations as clinical symptoms may be vague and clinical signs could be completely non-specific. We missed an underlying renal pathology in a paraplegic patient, who presented twice with loin swelling and redness. There was a delay in making a correct diagnosis because of our total reliance on the report of ultrasound scans, which failed to detect the perinephric lesion in this paraplegic patient with spinal curvature. We learn from this case that ultrasonography of kidney and perinephric region may not be completely reliable in SCI patients, who have marked curvature of spine. A joint team approach by health professionals belonging to various medical disciplines, which is strengthened by frequent, informal and honest discussions of a patient's clinical condition, is likely to reduce urological errors in SCI patients and improve the quality of their care.

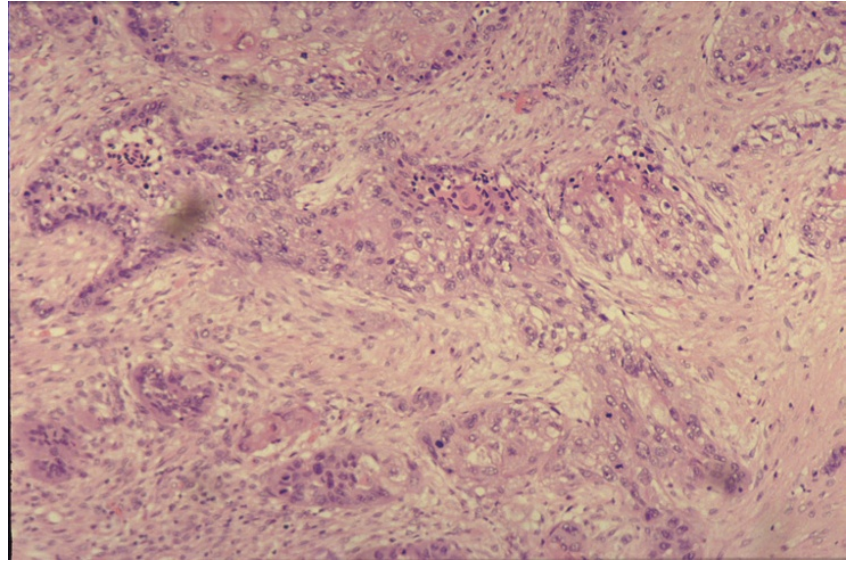

Figure 8

Photograph of repeat bladder biopsy in the same patient as in Figure 7. A second bladder biopsy was performed three weeks later. This biopsy revealed keratinising, moderately differentiated invasive, squamous cell carcinoma. (HPOI/ $07168)$

\section{Competing interests}

All authors are associated with the provision of medical care to SCI patients. Therefore, the authors sincerely wish to reduce medical errors in spinal cord injury patients.

\section{Acknowledgement}

We thank the patient for providing written consent for publication of the clinical photograph.

\section{References}

I. Strauther GR, Longo WE, Virgo KS, Johnson FE: Appendicitis in patients with previous spinal cord injury. Am J Surg 1999, 178:403405

2. Vaidyanathan S, Singh G, Soni BM, Hughes P, Watt JW, Dundas S, Sett $P$, Parsons KF: Silent hydronephrosis/pyonephrosis due to upper urinary tract calculi in spinal cord injury patients. Spinal Cord 2000, 38:661-668

3. Montgomery EA, Meis JM, Mitchell MS, Enzinger FM: Atypical decubital fibroplasia. A distinctive fibroblastic pseudotumor occurring in debilitated patients. Am J Surg Pathol 1992, 16:708-7I5

4. Saul N, Weingart SN, Ross McL, Wilson R, Robert W, Gibberd RW, Harrison B: Epidemiology of medical error BMJ 2000, 320:774777

5. Breatnach E, Stanley RJ, Bueschen AJ: CT demonstration of spontaneous extrusion of staghorn calculus J Comput Assist Tomogr 1986, 10:346

6. Institute of Medicine: To err is human: building a safety health system. Washington, DC, National Academy Press 1999

7. Navon JD, Soliman H, Khonsari F, Ahlering T: Screening cystoscopy and survival of spinal cord injured patients with squamous cell cancer of the bladder. J Urol 1997, 157:2109-21II

8. Vaidyanathan S, Mansour Paul, Soni BM, Singh G: Pathology of neuropathic bladder due to spinal cord injury. In: Pathology of the Urinary Bladder. Eds. CS Foster and JS Ross. Harcourt Health Sciences, New York, 